\title{
Study of the Interstellar Medium and Cosmic Rays in the MBM 53-55 Clouds and the Pegasus Loop
}

\author{
Tsunefumi Mizuno* on behalf of the Fermi-LAT Collaboration \\ Hiroshima Astrophysical Science Center, Hiroshima University, Higashi-Hiroshima, Hiroshima \\ 739-8526, Japan \\ E-mail: mizuno@astro.hiroshima-u.ac.jp
}

\begin{abstract}
High-energy cosmic-ray (CR) protons interact with the interstellar gas and produce intense diffuse $\gamma$ rays. Since the interstellar medium (ISM) is transparent to these high-energy photons, $\mathrm{GeV} \gamma$ rays are a powerful probe to study the ISM and Galactic CRs. In order to study the spatial and spectral distributions of CRs through $\gamma$ rays, either in the CR accelerators or in the interstellar space, the interstellar gas column densities need to be estimated with good accuracy using observations in other wavebands such as radio, infrared, and optical. Radio line observations at $21 \mathrm{~cm}$ and $2.6 \mathrm{~mm}$ have been used to trace the atomic and molecular gas distributions, respectively. Yet, a significant amount of gas not traced properly by these surveys was revealed by EGRET and has been confirmed by using Fermi Large Area Telescope (LAT) data. Although dust distribution has been used to trace these "dark gas", the procedure to convert dust observations into the total gas column density $\left(N\left(\mathrm{H}_{\text {tot }}\right)\right)$ has not been established yet. In this contribution, we report the study of ISM and CRs in the nearby molecular clouds MBM 53, 54, and 55 and a far-infrared loop-like structure in Pegasus. By combining the dust thermal emission model based on Planck data with Fermi-LAT $\gamma$-ray data, it was found that neither the dust radiance $(R)$ nor the dust opacity at $353 \mathrm{GHz}\left(\tau_{353}\right)$ was proportional to $N\left(\mathrm{H}_{\mathrm{tot}}\right)$, but instead, their ratios $\left(N\left(\mathrm{H}_{\mathrm{tot}}\right) / R\right.$ or $\left.N\left(\mathrm{H}_{\mathrm{tot}}\right) / \tau_{353}\right)$ depend on the dust temperature $T_{\mathrm{d}}$. Using the $\gamma$-ray data as a robust gas tracer to compensate for the temperature dependence, we evaluated $N\left(\mathrm{H}_{\text {tot }}\right)$ distributions by employing an empirical linear relation between $N\left(\mathrm{H}_{\text {tot }}\right) / R$ and $T_{\mathrm{d}}$. We present the data analysis, results, and implications for the ISM and CRs.
\end{abstract}

35th International Cosmic Ray Conference - ICRC2017

10-20 July, 2017

Bexco, Busan, Korea

${ }^{*}$ Speaker. 


\section{Introduction}

In the interstellar space, cosmic-ray (CR) protons interact with the interstellar gas and produce diffuse $\gamma$ rays. CR electrons also produce $\gamma$ rays but their contribution is smaller in GeV energy range with a typical proton-to-electron ratio. Because the interstellar medium (ISM) is essentially transparent to these high-energy photons, and the $\gamma$-ray production cross section is independent of the chemical or thermodynamic state of the interstellar gas, cosmic $\mathrm{GeV} \gamma$ rays have been recognized as a powerful probe to study the ISM and Galactic CRs. In order to study the spatial and spectral distributions of CRs through $\gamma$ rays, either in the CR accelerators or in the interstellar space, the interstellar gas column densities need to be estimated with good accuracy using observations in other wavebands such as radio, infrared, and optical.

Usually, the distribution of atomic hydrogen (HI) is measured by $21-\mathrm{cm}$ line surveys (e.g., [1]) and the distribution of molecular hydrogen $\left(\mathrm{H}_{2}\right)$ is derived via 2.6-mm line observations of carbon monoxide, $\mathrm{CO}$ (e.g., [2]). The total gas column density $\left(N\left(\mathrm{H}_{\mathrm{tot}}\right)\right)$ can also be estimated from extinction, reddening, or emission by dust (e.g., [3]). These tracers have advantages and disadvantages. Therefore, comparing H I, CO, and dust observations is crucial to study the interstellar gas distribution, and adding $\gamma$-ray data is important because it is another independent tracer of $N\left(\mathrm{H}_{\text {tot }}\right)$. Indeed, by combining the EGRET $\gamma$-ray data, $\mathrm{HI}, \mathrm{CO}$, and dust extinction maps, a significant amount of gas not traced by the H I or CO surveys was revealed in the solar neighborhood and has been referred to as "dark gas" [4]. This work has been confirmed and improved in terms of significance and accuracy by recent observations by Fermi-LAT (e.g., [5, 6]). Although the Planck satellite provides an accurate dust thermal emission model, which advances the study of the ISM (e.g., [7]), the procedure to convert the dust observations into the distribution of $N\left(\mathrm{H}_{\text {tot }}\right)$ has not been established yet. By comparing the Planck dust optical depth map at $353 \mathrm{GHz}\left(\tau_{353}\right)$ and the $\mathrm{HI} / \mathrm{CO}$ data, and assuming that $N\left(\mathrm{H}_{\mathrm{tot}}\right)$ was proportional to $\tau_{353}$, Fukui et al. [8, 9] proposed that a significant amount of the atomic hydrogen was optically thick in areas with low dust temperature $\left(T_{\mathrm{d}}\right)$. The Planck collaboration [10], on the other hand, found that the dust radiance $R$ (total dust emission integrated over frequency) was well correlated with the integrated HI $21-\mathrm{cm}$ line intensity, $W_{\mathrm{HI}}$, in wide range of $T_{\mathrm{d}}$ in the diffuse ISM, and proposed that it would be a better tracer of the dust (and the total gas) column density.

Here, we report an analysis of the Fermi-LAT $\gamma$-ray data in the Galactic longitudes $60^{\circ} \leq l \leq$ $120^{\circ}$ and the Galactic latitudes $-60^{\circ} \leq b \leq-28^{\circ}$. Our region of interest (ROI) encompasses the MBM 53, 54, and 55 molecular cloud complexes [11, 12] located at $l=84^{\circ}$ to $96^{\circ}$ and $b=-44^{\circ}$ to $-30^{\circ}$, and an infrared loop-like structure in Pegasus $[13,14]$ located in an area of $\sim 20^{\circ} \times 20^{\circ}$ around $(l, b) \sim\left(109^{\circ},-45^{\circ}\right)$. They are nearby $(100-150 \mathrm{pc})$ molecular clouds located at high Galactic latitudes (having small overlap with structures in the ISM at different distances), and therefore are expected to have uniform ISM and CR properties. Under the assumption of uniform CR density within the physical scale of the clouds, we examine the ratio of the $\gamma$-ray intensity associated with the ISM gas (i.e., a tracer of $\left.N\left(\mathrm{H}_{\text {tot }}\right)\right)$ to dust tracers $\left(R\right.$ or $\left.\tau_{353}\right)$, obtaine the disribution of $N\left(\mathrm{H}_{\text {tot }}\right)$ by using $\gamma$-ray data as a robust gas tracer, and discuss the properties of the ISM and CRs. In the following sections, we describe $\gamma$-ray modeling, data analysis, and discussion of the ISM and CR properties in Section 2, 3, and 4, respectively. Readers are also referred to [15] for more details. 


\section{Gamma-ray Data and Modeling}

\subsection{Gamma-ray observations and data selection}

The Large Area Telescope (LAT) on board the Fermi satellite is a pair-tracking $\gamma$-ray telescope, detecting photons from $\sim 20 \mathrm{MeV}$ to more than $300 \mathrm{GeV}[16,17]$. We have accumulated events from 2008 August 4 to 2015 August 2 to study diffuse $\gamma$ rays in our ROI. We used the Pass 8 event data, and selected events satisfying the Clean class using Fermi Science Tools ${ }^{1}$. We required that the reconstructed zenith angles of the arrival direction of the photons be less than $100^{\circ}$ to reduce contamination by photons from the Earth rim, and excluded the periods of time during which the LAT detected bright $\gamma$-ray bursts, solar flares, and flares of a bright active galactic nuclei 3C 454.3.

\subsection{Properties of the ISM tracers}

Because preparing good templates of the interstellar gas is crucial for $\gamma$-ray data analysis, we first investigated the properties of the ISM tracers in our ROI. We prepared dust maps, a $W_{\mathrm{HI}}$ map, and an integrated CO 2.6-mm line intensity ( $W_{\mathrm{CO}}$ ) map. We used the Planck dust maps (of $R, \tau_{353}$, and $T_{\mathrm{d}}$ ) of the public data release 1 (the version R1.20) ${ }^{2}$. As described by, e.g., [10], both $R$ and $\tau_{353}$ are expected to be proportional to $N\left(\mathrm{H}_{\mathrm{tot}}\right)$ to a first order approximation. To construct the $W_{\mathrm{HI}}$ map, we referred to the Leiden/Argentine/Bonn (LAB) survey [18]. The correlations between $W_{\mathrm{HI}}$ and $R$, and those between $W_{\mathrm{HI}}$ and $\tau_{353}$, are shown in Figure 1. To exclude the regions of $\mathrm{H}_{2}$ associated with appreciable $W_{\mathrm{CO}}$, we referred to a $W_{\mathrm{CO}}$ map internally available to the LAT team, which is based on the work by [2], and masked areas with $W_{\mathrm{CO}}$ intensity greater than $1.1 \mathrm{~K} \mathrm{~km} \mathrm{~s}^{-1}$. We can confirm the trends of the dust-gas relation found by previous studies as (1) we observe in Figure 1 (left) a good correlation between the $W_{\mathrm{HI}}$ and $R$ in a wide range of $T_{\mathrm{d}}$ [10] and (2) we observe in Figure 1 (right) a strong $T_{\mathrm{d}}$ dependence of the $W_{\mathrm{HI}}-\tau_{353}$ relation $[8,9]$. The correlation between the dust tracers and $W_{\mathrm{H}_{\mathrm{I}}}$ alone is not sufficient to distinguish which $\left(R\right.$ or $\left.\tau_{353}\right)$ is the better tracer of the total dust (and gas) column density. We therefore prepared two types of $N\left(\mathrm{H}_{\text {tot }}\right)$ model maps based on $R$ and $\tau_{353}$ and tested them against the Fermi-LAT $\gamma$-ray data as described in Section 3.
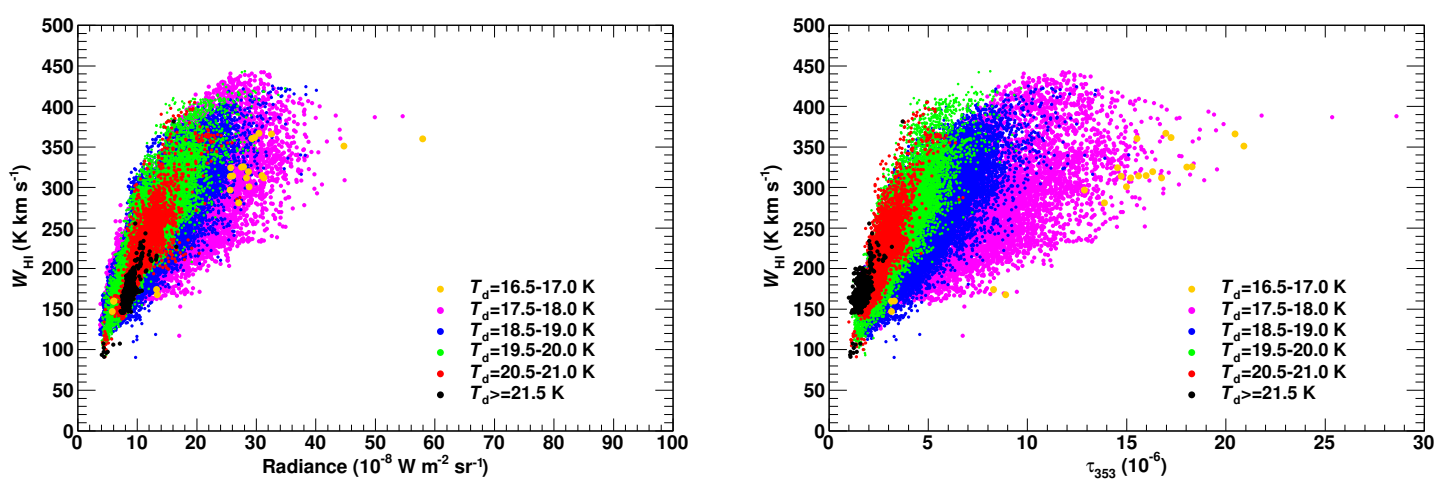

Figure 1: The correlations between $W_{\mathrm{HI}}$ and dust tracers: (left) scatter plot of $W_{\mathrm{HI}}$ versus $R$ and (right) scatter plot of $W_{\mathrm{HI}}$ versus $\tau_{353}$. (shown in [15], reproduced courtesy of the AAS)

\footnotetext{
${ }^{1}$ http://fermi.nasa.gov/ssc/data/analysis/software/

${ }^{2}$ http://irsa.ipac.caltech.edu/data/Planck/release_1/all-sky-maps/
} 


\subsection{Model to represent the gamma-ray emission}

We modeled the $\gamma$-ray emission as a linear combination of the gas column density model map(s) constructed from the Planck observations, inverse Compton (IC) emission model, isotropic component and $\gamma$-ray point sources. The use of the gas column density maps as a template is based on the assumption that $\gamma$ rays are generated via interactions between the CRs and ISM gas and that CR densities do not vary significantly over the ROI. We started with a single $N\left(\mathrm{H}_{\text {tot }}\right)$ map based on $R$ or $\tau_{353}$, and employed multiple $N\left(\mathrm{H}_{\mathrm{tot}}\right)$ maps sorted by $T_{\mathrm{d}}$ in Section 3.1. To model the $\gamma$ rays produced via IC scattering, we adopted the IC model map produced in the GALPROP 3 (e.g., $[19,20]$ ) run 54_77Xvarh7S as our baseline model but multiplied an energy-dependent scale factor as a free parameter to accomodate the uncertainties of the spectrum. To model the individual $\gamma$-ray sources, we referred to the third Fermi-LAT catalog (3FGL) described in [21] and included 57 (detected at a significance of $\geq 5 \sigma)$ in our ROI, and 17 bright sources $(\geq 20 \sigma)$ just outside it (within $10^{\circ}$ ) to take account of their possible contamination. To accomodate the possible change of the spectrum, we adopted the position of sources given by [21] but set the normalization free in each energy band for the analysis. An isotropic component is to represent the extragalactic diffuse $\gamma$-ray emission and the residual instrumental background. Then, $\gamma$-ray intensities $I_{\gamma}(l, b, E)\left(\mathrm{ph} \mathrm{s}^{-1} \mathrm{~cm}^{-2} \mathrm{sr}^{-1} \mathrm{MeV}^{-1}\right)$ can be modeled as

$I_{\gamma}(l, b, E)=\sum_{i} c_{1, i}(E) \cdot q_{\gamma}(E) \cdot N\left(\mathrm{H}_{\mathrm{tot}}\right)_{i}(l, b)+c_{2}(E) \cdot I_{\mathrm{IC}}(l, b, E)+I_{\mathrm{iso}}(E)+\sum_{j} c_{3, j}(E) \cdot \operatorname{PS}_{j}(l, b)$,

where $N\left(\mathrm{H}_{\mathrm{tot}}\right)_{i}$ is the total gas column density model $\left(\mathrm{cm}^{-2}\right) \operatorname{map}(\mathrm{s}), c_{1, i}(E) \cdot q_{\gamma}(E)\left(\mathrm{ph} \mathrm{s}^{-1} \mathrm{sr}^{-1} \mathrm{MeV}^{-1}\right)$ is the differential $\gamma$-ray emissivity per $\mathrm{H}$ atom, $c_{2}(E) \cdot I_{\mathrm{IC}}(l, b, E)$ and $I_{\text {iso }}(E)$ are the IC model and isotropic background intensities, respectively, and $c_{3, j}(E) \cdot \mathrm{PS}_{j}(l, b)$ represents the point source contributions. The subscript $i$ allows for the separation of $N\left(\mathrm{H}_{\mathrm{tot}}\right)$ maps by $T_{\mathrm{d}}$ (Section 3.1). $q_{\gamma}(E)$ is the $\gamma$-ray emissivity model for the local interstellar spectrum (LIS) of CRs and the nuclear enhancement factor $\varepsilon_{\mathrm{M}}$ (a scale factor to take account of the effect of heavy nuclei in both CRs and the target matter) of 1.84 [22] adopted by [23].

\section{Data Analysis}

To investigate the temperature dependence we observed in Section 2.2 more quantitatively, we used $\gamma$-ray as a robust tracer of $N\left(\mathrm{H}_{\text {tot }}\right)$ as described below.

\subsection{Dust temperature-sorted modeling}

We started our data analysis using a single $N\left(\mathrm{H}_{\mathrm{tot}}\right)$ model model maps based on $R$ or $\tau_{353}$. To construct the model maps, we assumed a proportionality between $N\left(\mathrm{H}_{\mathrm{tot}}\right)$ and $R\left(\right.$ or $\left.\tau_{353}\right)$ and that $\mathrm{HI}$ is optically thin and well represents the total gas column density at least for regions with the high-temperature areas. We made least-squares fit to the $T_{\mathrm{d}} \geq 21.5 \mathrm{~K}$ dust- $W_{\mathrm{HI}}$ relation in Figure 1 with a linear function and obtained coefficients of $19.8 \times 10^{8} \mathrm{~K} \mathrm{~km} \mathrm{~s}^{-1}\left(\mathrm{~W} \mathrm{~m}^{-2} \mathrm{sr}^{-1}\right)^{-1}$ and $102 \times 10^{6} \mathrm{~K} \mathrm{~km} \mathrm{~s}^{-1}$ for the $R$ and $\tau_{353}$, respectively. We then converted $R$ (or $\tau_{353}$ ) into $N\left(\mathrm{H}_{\text {tot }}\right.$ ) maps, using the coefficients obtained and multiplied by $1.82 \times 10^{18} \mathrm{~cm}^{-2}\left(\mathrm{~K} \mathrm{~km} \mathrm{~s}^{-1}\right)^{-1}$. The

\footnotetext{
$3_{\text {http: }}$ //galprop.stanford.edu
} 
model described in Equation (2.1) was fitted to the data from 0.3 to $72.9 \mathrm{GeV}$ divided into several energy bands. We then split the $N\left(\mathrm{H}_{\text {tot }}\right)$ template map (constructed from $R$ or $\tau_{353}$ ) into four based on $T_{\mathrm{d}}$, for $T_{\mathrm{d}} \leq 18 \mathrm{~K}, T_{\mathrm{d}}=18-19 \mathrm{~K}, T_{\mathrm{d}}=19-20 \mathrm{~K}$ and $T_{\mathrm{d}} \geq 20 \mathrm{~K}$ and fit $\gamma$-ray data with Equation (2.1) using the four template maps, with scaling factors $\left(c_{1, i}(E)\right)$ freely varying individually. We calculated the $\log$-likelihoods $(\ln L)$ and confirmed that the fit improvement was significant in both $R$-based and $\tau_{353}$-based cases.

If either $R$ or $\tau_{353}$ is proportional to $N\left(\mathrm{H}_{\mathrm{tot}}\right)$, the scaling factors $c_{1, i}(E)$ are expected to be independent on $T_{\mathrm{d}}$. However, we observed that they depended on $T_{\mathrm{d}}$; the averages over the entire energy range are summarized in Figure 2 (left) which shows clear correlations for both $R$-based and $\tau_{353}$-based $N\left(\mathrm{H}_{\mathrm{tot}}\right)$ maps. These trends cannot be interpreted as being due to the properties of CRs, because the physical environments that determine the $T_{\mathrm{d}}$ (e.g., the interstellar radiation field intensity and dust cross section) do not affect the CR density.

In order to examine if the $T_{\mathrm{d}}$ dependence we found is an artifact due to the systematic uncertainty of our modeling, we carried out several tests as the followings. (1) We tested several other IC models having different spatial distributions. (2) We masked areas around the dense molecular clouds traced by $\mathrm{CO}$, since $N\left(\mathrm{H}_{\mathrm{tot}}\right) / R$ or $N\left(\mathrm{H}_{\mathrm{tot}}\right) / \tau_{353}$ could be appreciably different in dense molecular cloud complexes from that in translucent clouds. (3) We also masked intermediatevelocity clouds [24] in our ROI since they could have different CR density and/or different ISM gas and dust properties. We confirmed that the $T_{\mathrm{d}}$ dependence we found is robust against these tests, and gauged systematic uncertainties by bracketing the $T_{\mathrm{d}}$ dependence of our baseline model as shown by the shaded bands in Figure 2 (left). We thus found that $N\left(\mathrm{H}_{\mathrm{tot}}\right) / R$ and $N\left(\mathrm{H}_{\mathrm{tot}}\right) / \tau_{353}$ (both $\propto c_{1, i}$ ) are not uniform and depend on $T_{\mathrm{d}}$ in our ROI by using $\gamma$-ray data as a robust gas tracer.
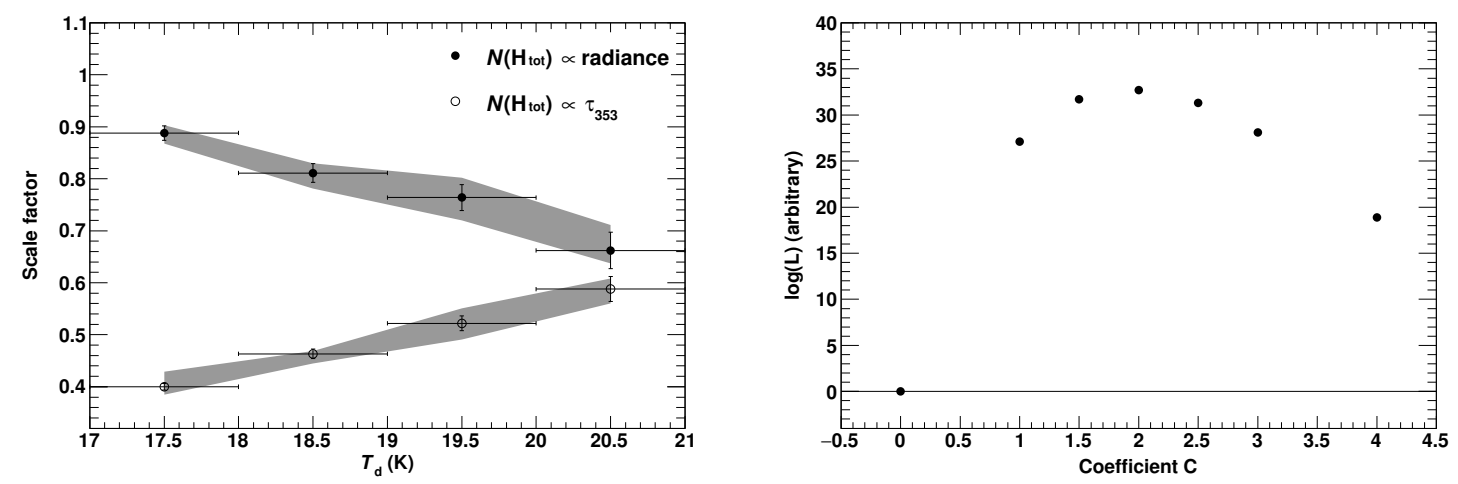

Figure 2: (left) Summary of the scale factors $c_{1, i}$ in Equation (2.1) averaged over the entire energy range in each range of $T_{\mathrm{d}}$ with the systematic uncertainty shown by gray bands. (right) Summary of $\ln L$ as a function of the coefficient $C$ in Equation (3.1) with $T_{\mathrm{bk}}=20.5 \mathrm{~K}$. (shown in [15], reproduced courtesy of the AAS)

\subsection{Dust temperature-corrected modeling}

As described in Section 3.1, we found the $T_{\mathrm{d}}$ dependence of the scaling factors of the gas component and concluded that it was primarily due to the dust properties. We aim to use the $\gamma$-ray data as a robust tracer of $N\left(\mathrm{H}_{\text {tot }}\right)$ and apply a correction on the $N\left(\mathrm{H}_{\text {tot }}\right)$ model map to compensate for $T_{\mathrm{d}}$ dependence. 
We started from the $R$-based single $N\left(\mathrm{H}_{\text {tot }}\right)$ map and modified the gas column density with an empirical function as below:

$$
N\left(\mathrm{H}_{\mathrm{tot}, \mathrm{mod}}\right)=\left\{\begin{array}{l}
N\left(\mathrm{H}_{\mathrm{tot}, \mathrm{R}}\right)\left(T_{\mathrm{d}}>T_{\mathrm{bk}}\right), \\
\left(1+0.05 \cdot C \cdot \frac{T_{\mathrm{bk}}-T_{\mathrm{d}}}{1 \mathrm{~K}}\right) \cdot N\left(\mathrm{H}_{\mathrm{tot}, \mathrm{R}}\right)\left(T_{\mathrm{d}} \leq T_{\mathrm{bk}}\right),
\end{array}\right.
$$

where $T_{\mathrm{bk}}=20.5 \mathrm{~K}$. Above $T_{\mathrm{bk}}$ we retained the original $N\left(\mathrm{H}_{\mathrm{tot}}\right)$ distribution. We re-examined the correlation between $R$ and $W_{\mathrm{HI}}$ (Figure 1 (left)) and confirmed that the coefficient between the two quantities, originally determined for $T_{\mathrm{d}}$ above $21.5 \mathrm{~K}$, was unchanged above $20.5 \mathrm{~K}$. Therefore, our procedure is self-consistent. We tested several choices of coefficients $C$ and summarized the value of $\ln L$ in Figure 2 (right). We found that $C$ of 2 (which corresponds to a $10 \%$ required increase in the gas column density as $T_{\mathrm{d}}$ decreases by $1 \mathrm{~K}$ ) gave the best fit to the Fermi-LAT data. The obtained $N\left(\mathrm{H}_{\text {tot,mod }}\right)$ map together with the data and model count map is shown in Figure 3. Below we discuss the properties of the ISM and CRs in the region studied using the $N\left(\mathrm{H}_{\mathrm{tot}, \mathrm{mod}}\right)$ map.
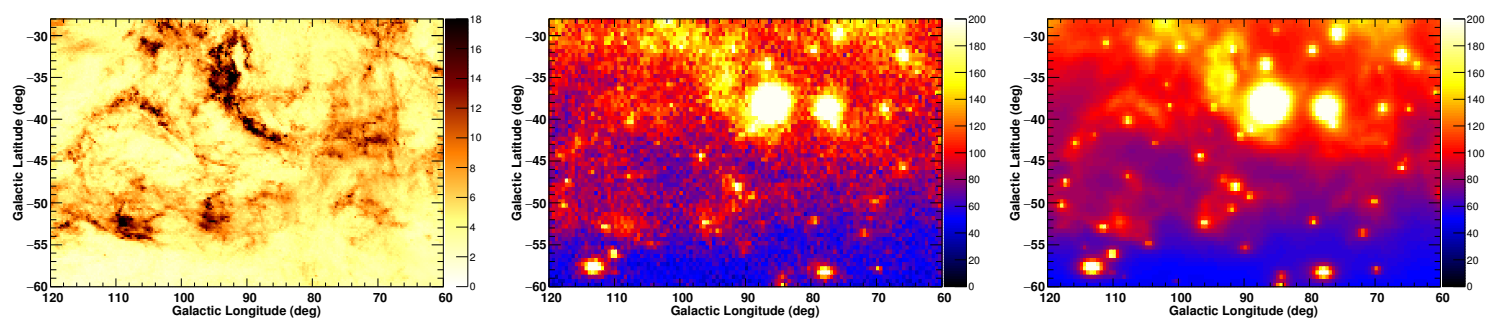

Figure 3: (left) The corrected $N\left(\mathrm{H}_{\mathrm{tot}}\right)$ map in unit of $10^{20} \mathrm{~cm}^{-2}$, and (middle) the data count map, and (right) the model count map. (shown in [15], reproduced courtesy of the AAS)

\section{Discussion}

\subsection{ISM gas distribution}

Because the molecular clouds studied here are located at similar distances from the solar system and most of the HI clouds are expected to coexist with them (because they are located at high Galactic latitudes), we can estimate the mass of gas from the column density $N(\mathrm{H})$ as

$$
M=\mu m_{\mathrm{H}} d^{2} \int N(\mathrm{H}) d \Omega,
$$

where $d$ is the distance to the cloud, $m_{\mathrm{H}}$ is the mass of the hydrogen atom and $\mu=1.41$ is the mean atomic mass per $\mathrm{H}$ atom [25]. From Equation (4.1), $\int N(\mathrm{H}) d \Omega=10^{22} \mathrm{~cm}^{-2} \mathrm{deg}^{2}$ corresponds to $\sim 740 \mathrm{M}_{\odot}$ for $d=150 \mathrm{pc}$. The integrated H I column density for the optically thin case, $\int N\left(\mathrm{HI}_{\mathrm{thin}}\right) d \Omega=1.82 \times 10^{18} \cdot \int W_{\mathrm{HI}} d \Omega$, and the integrated column density of the excess gas $\left(\int\left(N\left(\mathrm{H}_{\text {tot }}\right)-N\left(\mathrm{HI}_{\mathrm{thin}}\right)\right) d \Omega\right)$ are shown in Figure 4 (left). We observed that the excess gas starts to appear below $T_{\mathrm{d}}=20 \mathrm{~K}$ and is as massive as that of $\mathrm{HI}$ for the optically thin case below $18 \mathrm{~K}$.

Some fraction of this excess gas is molecular hydrogen traced by $\mathrm{CO}$ (hereafter denoted as $\mathrm{H}_{2, \mathrm{CO}}$ ), and we can calculate the mass as

$$
M=\mu m_{\mathrm{H}} d^{2} \int 2 N\left(\mathrm{H}_{2, \mathrm{CO}}\right) d \Omega=\mu m_{\mathrm{H}} d^{2} \cdot 2 X_{\mathrm{CO}} \int W_{\mathrm{CO}} d \Omega .
$$


Assuming $X_{\mathrm{CO}}=1.25 \times 10^{20} \mathrm{~cm}^{-2}\left(\mathrm{~K} \mathrm{~km} \mathrm{~s}^{-1}\right)^{-1}$, we calculated $2 X_{\mathrm{CO}} \int W_{\mathrm{CO}} d \Omega$ as a measure of the CO-bright $\mathrm{H}_{2}$ mass and plotted the distribution in Figure 4 (left). The integral of $N\left(\mathrm{HI}_{\text {thin }}\right)$ and $N\left(\mathrm{H}_{\mathrm{tot}}\right)-N\left(\mathrm{HI}_{\mathrm{thin}}\right)$ is 60.9 and 16.5 in units of $10^{22} \mathrm{~cm}^{-2} \mathrm{deg}^{2}$, respectively, and that of $W_{\mathrm{CO}}$ multiplied by $2 X_{\mathrm{CO}}$ is 2.6 in the same units. As shown in Figure 4 (left), the contribution of the CO-bright molecular mass is $\leq 10 \%$ at $T_{\mathrm{d}}$ above $17.5 \mathrm{~K}$. This is the "dark gas" and it contributes $(16.5-2.6) / 60.9 \sim 25 \%$ of the mass in $\mathrm{HI}$ in the optically thin case.

The ratio of the mass of dark gas to that of the $\mathrm{H}_{2}$ traced by $\mathrm{CO}$ is $(16.5-2.6) / 2.6 \sim 5$, significantly higher than the values in other regions obtained by Fermi-LAT (e.g., [7, 26]). In other words, the region studied here is dark-gas-rich when compared to $\mathrm{H}_{2}$ traced by $\mathrm{CO}$. If we use the $R$-based and $\tau_{353}$-based $N\left(\mathrm{H}_{\mathrm{tot}}\right)$ maps uncorrected for the dust temperature effect, the integral of $N\left(\mathrm{H}_{\text {tot }}\right)-N\left(\mathrm{HI}_{\text {thin }}\right)$ is 6.2 and 56.8 in units of $10^{22} \mathrm{~cm}^{-2} \mathrm{deg}^{2}$, respectively, whereas we obtained 16.5 in the same units through $\gamma$-ray data analysis. Then, the dark gas contribution (obtained by subtracting $2 X_{\mathrm{CO}} \int W_{\mathrm{CO}} d \Omega$ estimated to be $\sim 2.6$ ) based on uncorrected $R$-based and $\tau_{353}$-based $N\left(\mathrm{H}_{\text {tot }}\right)$ maps will be 3.6 and 54.2, respectively, and is a factor of $\sim 4$ lower/higher than what we found $(16.5-2.6=13.9)$; therefore, the correction based on the $\gamma$-ray analysis is crucial. We also note that the required correction we found is the average of the studied region. A systematic study of other high-latitude regions by Fermi-LAT in combination with the Planck dust model is required to examine the uniformity/variation of the ISM properties in the solar neighborhood, which will be key ingredient for understanding the nature of the dark gas (e.g., optically-thick H I $[8,9]$ or CO-dark $\mathrm{H}_{2}$ [27]).

\subsection{CR density}

Finally, we discuss the HI emissivity spectrum obtained in this study shown in Figure 4 (right). To examine the systematic uncertainty, we repeated the same analysis in Section 3.1 and evaluated the variation of $W_{\mathrm{HI}_{\mathrm{I}}}-\mathrm{to}-R$ by dividing the region with $T_{\mathrm{d}} \geq 20.5 \mathrm{~K}$ into six sub-regions. The obtained total systematic uncertainty is shown by the shaded band. For comparison, we plotted the model curves for the LIS that we adopted and $\varepsilon_{\mathrm{M}}$ of 1.84 and 1.45 (the lowest value referred to in [22]) in the same figure. We also plotted the emissivity spectrum of the local H I clouds measured by [23] and [28] for comparison. A significant difference can be seen in the normalization. While our result agrees with the model with $\varepsilon_{\mathrm{M}}=1.45$, relevant studies by Fermi-LAT favor the model with $\varepsilon_{\mathrm{M}}=1.85$. This difference can be understood, at least partially, due to the assumption of the H I optical thickness. [23] and [28] assumed a uniform spin temperature $T_{\mathrm{S}}$ of $125 \mathrm{~K}$ and $140 \mathrm{~K}$, respectively. If uniform $T_{\mathrm{S}}$ of such values is applied to our region, we will have smaller $N\left(\mathrm{H}_{\mathrm{tot}}\right)$ than that we obtained and larger HI emissivity.

More specifically, the difference comes from different assumptions about gas and dust properties. The method presented here relies on the uniformity of $N\left(\mathrm{H}_{\mathrm{tot}}\right) / R$ for the same value of $T_{\mathrm{d}}$ regardless of the gas phases, while a conventional template-fitting method relies on the uniformity of $T_{\mathrm{s}}, X_{\mathrm{CO}}$, and $N(\mathrm{H}) / D$ (where $D$ is the employed dust map such as $R$ and $\tau_{353}$ ) in each gas phase across the ROI. Therefore using a good dust tracer is crucial for the conventional method, and several alternative tracers of dust need to be compared (e.g., [26]). The method developed here, on the other hand, has more flexibility to adjust $N\left(\mathrm{H}_{\text {tot }}\right) / D$, and implies lower $\mathrm{H}$ I emissivity due to, e.g., lower fraction of heavy CR nuclei and/or smaller cross sections other than for $\mathrm{p}-\mathrm{p}$ collisions than previously inferred from gas emissivities in $\gamma$ rays. 
Although our analysis was based on a few basic assumptions on properties of the gas, dust, and CRs, and we have carefully examined the systematic uncertainty, the method is still in an early phase of development and should be tested and improved by applying to other regions of the sky. In such future analyses, a comparison with the conventional method should be done at the same time to examine the merits and demerits of each method and the validity of the underlying assumptions, and to better understand the properties of the ISM and CRs in the solar neighborhood.
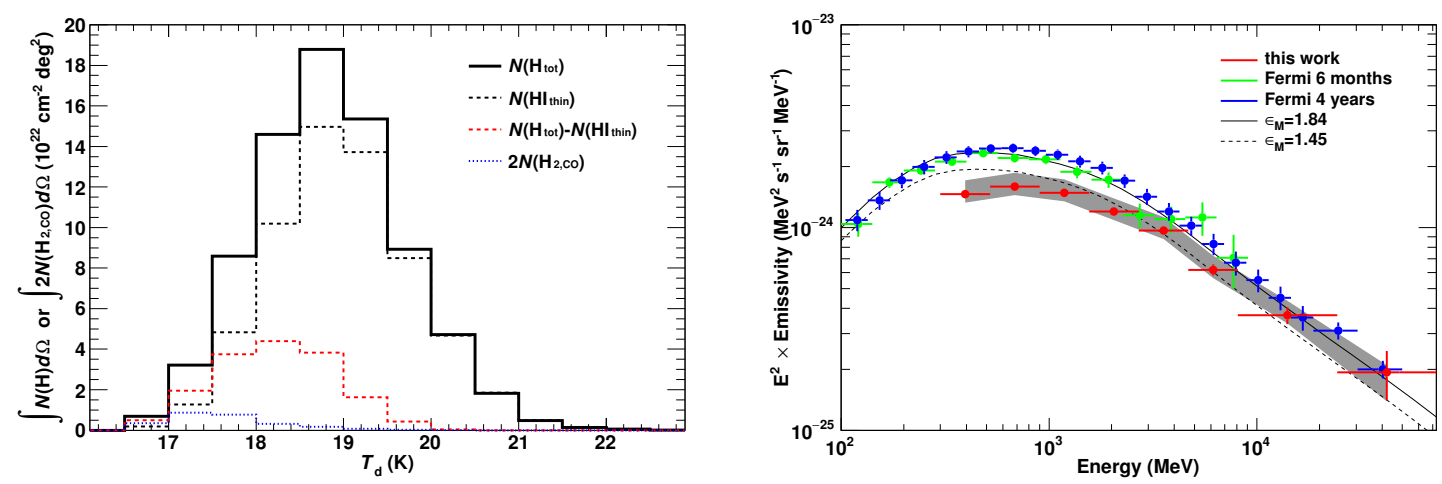

Figure 4: (left) The distribution of the integrated HI column density for the optically thin case, $\int N\left(\mathrm{HI}_{\mathrm{thin}}\right) d \Omega=1.82 \times 10^{18} \cdot \int W_{\mathrm{HI}} d \Omega$, and that of the integrated excess gas column density above $N\left(\mathrm{HI}_{\text {thin }}\right)$. The integrated $W_{\mathrm{CO}}$ as a measure of the CO-bright $\mathrm{H}_{2}$ is also plotted. (right) Summary of the $\mathrm{HI}$ emissivity spectrum obtained in this study compared with the model curves based on the LIS for $\varepsilon_{\mathrm{M}}=$ 1.45 and 1.84, and the results of the relevant studies by Fermi-LAT [23, 28]. The shaded band shows the systematic uncertainty of our H I emissivity spectrum. (shown in [15], reproduced courtesy of the AAS)

The Fermi-LAT Collaboration acknowledges support for LAT development, operation and data analysis from NASA and DOE (United States), CEA/Irfu and IN2P3/CNRS (France), ASI and INFN (Italy), MEXT, KEK, and JAXA (Japan), and the K.A. Wallenberg Foundation, the Swedish Research Council and the National Space Board (Sweden). Science analysis support in the operations phase from INAF (Italy) and CNES (France) is also gratefully acknowledged. This work performed in part under DOE Contract DE-AC02-76SF00515.

\section{References}

[1] Dickey, J. M., \& Lockman, F. J. 1990, ARA\&A, 28, 215

[2] Dame, T. M. et al. 2001, ApJ, 547, 792

[3] Bohlin, R. C. et al. 1978, ApJ, 224, 132

[4] Grenier, I. A. et al. 2005, Science, 307, 1292

[5] Abdo, A. A. et al. 2010, ApJ, 710, 133

[6] Ackermann, M. et al. 2012b, ApJ, 755, 22

[7] Planck Collaboration XXIV 2011, A\&A, 536, 24

[8] Fukui, Y. et al. 2014, ApJ, 796, 59

[9] Fukui, Y. et al. 2015, ApJ, 798, 6

[10] Planck Collaboration XI 2014, A\&A, 571, 11

[11] Yamamoto, H. et al. 2003, ApJ, 592, 217

[12] Welty, D. E et al. 1989, ApJ, 346, 232

[13] Kiss, Cs. et al. 2004, A\&A, 418, 131

[14] Yamamoto, H. et al. 2006, ApJ, 642, 307
[15] Mizuno, T. et al. 2016, ApJ 833, 278

[16] Atwood, W. B. et al. 2009, ApJ, 697, 1071

[17] Abdo, A. A. et al. 2009a, Astropart. Phys., 32, 193

[18] Kalberla, P. M. W. et al. 2005, A\&A, 440, 775

[19] Strong, A. W., \& Moskalenko, I., 1998, ApJ, 509, 212

[20] Strong, A. W. et al. 2007, ARA\&A, 57, 285

[21] Acero, F. et al., 2015, ApJS, 23, 41

[22] Mori, K. 2009, Astropart.Phys., 31, 341

[23] Abdo, A. A. et al. 2009b, ApJ, 703, 1249

[24] Wakker, B. P. 2001, ApJS, 136, 463

[25] Däppen, W. 2000, in Allen's Astrophysical Quantities, ed. A. N. Cox (4th ed.; New York: Springer), 27

[26] Planck Collaboration XXVIII 2015, A\&A, 582, 31

[27] Wolfire, M. G. et al. 2010, ApJ, 716, 1191

[28] Casandjian, J.-M. 2015, ApJ, 806, 240 